PROCEEDINGS OF THE

AMERICAN MATHEMATICAL SOCIETY

Volume 128, Number 6, Pages 1729-1732

S 0002-9939(99)05228-4

Article electronically published on October 29, 1999

\title{
THE LU QI-KENG CONJECTURE FAILS FOR STRONGLY CONVEX ALGEBRAIC COMPLETE REINHARDT DOMAINS IN $\mathbb{C}^{n}(n \geq 3)$
}

\author{
NGUYÊN VIÊT ANH
}

(Communicated by Steven R. Bell)

\begin{abstract}
In this note, we give an example of a strongly convex algebraic complete Reinhardt domain which is not Lu Qi-Keng in $\mathbb{C}^{n}$ for any $n \geq 3$.
\end{abstract}

\section{IntRoduCtion AND STATEMENT OF THE MAIN RESUlt}

Let us first recall that a domain $\Omega$ in $\mathbb{C}^{n}$ is said to be Lu Qi-Keng if its Bergman kernel function $K_{\Omega}(z, w)$ is non zero at any point $(z, w) \in \Omega \times \Omega$. The question of finding smooth pseudoconvex domains in $\mathbb{C}^{n}$ whose Bergman kernel function has zeros was raised by Lu Qi-Keng $[\mathrm{Lu}$. It is well-known [SY] that when $n=1$, then a smooth domain $\Omega$ in $\mathbb{C}$ is Lu Qi-Keng if and only if it is simply connected. For a complete history of this subject, see [Bo1], Bo2. In higher dimensions, Boas, $\mathrm{Fu}$ and Straube [BFS] proved that, for any $n \geq 3$, there exists a strongly convex real analytic domain in $\mathbb{C}^{n}$ which is not Lu Qi-Keng. Their example is deduced by exhaustion from the Hartogs triangle in $\mathbb{C}^{n}$. Recently, P. Pflug and E. H. Youssfi $[\mathrm{PY}]$ proved that the minimal balls defined in $[\mathrm{HP}$ are non Lu Qi-Keng for any $n \geq 4$. As a consequence, they gave concrete strongly convex algebraic and $S^{1} \cdot S O(n, \mathbb{R})$-invariant non Lu Qi-Keng balls in $\mathbb{C}^{n}$ for any $n \geq 4$, but these balls are not Reinhardt. In this note, we shall give concrete strongly convex algebraic complete Reinhardt domains which are non Lu Qi-Keng in $\mathbb{C}^{n}$ for any $n \geq 3$. This completes the works in [PY] and [BFS].

Consider, for a positive integer $n$, a non-negative integer $m$ and a real number $a \in] 0,1]$, the domain

$$
\Omega_{a, k, n, m}:=\left\{z=\left(z_{1}, z_{2}, \ldots, z_{n+m}\right) \in \mathbb{C}^{n+m}: N_{a, k}(z)<1\right\},
$$

where

$$
N_{a, k}(z):=\left(\sum_{\epsilon_{1}, \ldots, \epsilon_{n+1} \in\{-1,+1\}} \alpha_{\epsilon_{1}, \ldots, \epsilon_{n+1}}^{2 k}(z)+a^{2 k} \alpha^{2 k}(z)\right)^{\frac{1}{2 k}}
$$

Received by the editors July 14, 1998.

1991 Mathematics Subject Classification. Primary 32H10.

Key words and phrases. Lu Qi-Keng conjecture, Bergman kernel.

(C)2000 American Mathematical Society 


$$
\begin{aligned}
\alpha_{\epsilon_{1}, \ldots, \epsilon_{n+1}}(z) & :=\sum_{j=1}^{n} \epsilon_{j}\left|z_{j}\right|+\epsilon_{n+1} \cdot\left(\sum_{j=1}^{m}\left|z_{n+j}\right|^{2}\right), \\
\alpha(z) & :=\sum_{j=1}^{n+m}\left|z_{j}\right|^{2} .
\end{aligned}
$$

Our main result is the following

Theorem. Suppose that $a \in] 0,1], n$ is a positive integer and $m$ is a non-negative integer. Then

(1) the domain $\Omega_{a, k, n, m}$ is strongly convex, algebraic, complete Reinhardt;

(2) if $2 n+m>4$, then there exists a positive integer $M=M(a, n, m)$ such that $\forall k \geq M$, the domain $\Omega_{a, k, n, m}$ is non Lu Qi-Keng.

Remark. If we take $m=0$, then from the above theorem we see that there are strongly convex algebraic complete Reinhardt domains which are non Lu Qi-Keng in $\mathbb{C}^{n}$ for any $n \geq 3$.

\section{Preparatory Results and the proof of the main Result}

Let $D_{n, m}$ be the complex ellipsoid in $\mathbb{C}^{n+m}$ defined by:

$$
D_{n, m}:=\left\{z \in \mathbb{C}^{n+m}: \sum_{j=1}^{n}\left|z_{j}\right|+\sum_{j=n+1}^{m}\left|z_{j}\right|^{2}<1\right\} .
$$

This is a convex complete Reinhardt domain. From the recent work in [BFS], we have the following

Lemma 1.1. The domain $D_{n, m}$ is non Lu Qi-Keng if and only if $2 n+m>4$.

Proof. The case $2 n+m>4$ is a consequence of the principle of deflation in BFS]. The remaining case $2 n+m \leq 4$ was already treated in [BFS].

Lemma 1.2. The function $f$ of two real variables $(x, y)$ defined by $f(x, y):=$ $(x+y)^{2 k}+(x-y)^{2 k}$, where $k$ is a positive integer, is convex.

Proof. A little computing shows that the Hessian form [HLP, p. 80]

$$
\frac{\partial^{2} f}{\partial^{2} x} u^{2}+2 \frac{\partial^{2} f}{\partial x \partial y} u v+\frac{\partial^{2} f}{\partial^{2} y} v^{2} \geq 0, \forall(u, v) \in \mathbb{R}^{2} .
$$

Lemma 1.3. Consider $p$ convex functions

$$
f_{1}, \ldots, f_{p}: \mathbb{R}^{q} \rightarrow \mathbb{R}^{+}, \quad q \text { is a positive integer. }
$$

Then the function $\rho(z):=\sum_{\epsilon_{1}, \ldots, \epsilon_{p} \in\{-1,+1\}}\left(\epsilon_{1} f_{1}(z)+\cdots+\epsilon_{p} f_{p}(z)\right)^{2 k}$, where $k$ is a positive integer, is convex.

Proof. Consider $z, w \in \mathbb{R}^{q}$. By virtue of Lemma 1.2, we obtain easily that

$$
\frac{\rho(z)+\rho(w)}{2} \geq \sum_{\epsilon_{1}, \ldots, \epsilon_{p} \in\{-1,+1\}}\left(\epsilon_{1} \frac{f_{1}(z)+f_{1}(w)}{2}+\cdots+\epsilon_{p} \frac{f_{p}(z)+f_{p}(w)}{2}\right)^{2 k} .
$$


Using multi-nomial expansion of the expression $\left(\epsilon_{1} a_{1}+\cdots+\epsilon_{p} a_{p}\right)^{2 k}$ with $\epsilon_{1}, \ldots, \epsilon_{p}$ $\in\{-1,+1\}$, we get the identity

$$
\sum_{\epsilon_{1}, \ldots, \epsilon_{p} \in\{-1,+1\}}\left(\epsilon_{1} a_{1}+\cdots+\epsilon_{p} a_{p}\right)^{2 k}=2^{p} \cdot \sum_{k_{1}+\cdots+k_{p}=k} \frac{(2 k) ! a_{1}^{2 k_{1}} \ldots a_{p}^{2 k_{p}}}{\left(2 k_{1}\right) ! \ldots\left(2 k_{p}\right) !} .
$$

Combining (1.2) with (1.3), we obtain

$$
\begin{aligned}
\frac{\rho(z)+\rho(w)}{2} & \geq \sum_{k_{1}+\cdots+k_{p}=k} \frac{2^{p}(2 k) !}{\left(2 k_{1}\right) ! \ldots\left(2 k_{p}\right) !} \cdot\left(\frac{f_{1}(z)+f_{1}(w)}{2}\right)^{2 k_{1}} \\
& \ldots\left(\frac{f_{p}(z)+f_{p}(w)}{2}\right)^{2 k_{p}} .
\end{aligned}
$$

Using the hypothesis that the functions $f_{1}, \ldots, f_{p}$ are convex, positive and identity (1.3), we deduce that $\frac{\rho(z)+\rho(w)}{2} \geq \rho\left(\frac{z+w}{2}\right)$.

Proof of the main theorem. We shall use the defining function

$$
\rho(z):=\sum_{\epsilon_{1}, \ldots, \epsilon_{n+1} \in\{-1,+1\}} \alpha_{\epsilon_{1}, \ldots, \epsilon_{n+1}}^{2 k}(z)+a^{2 k} \alpha^{2 k}(z)-1 \quad \text { for } \Omega_{a, k, n, m} .
$$

Using identity (1.3), we see that $\rho(z)$ is a polynomial with positive coefficients in $\left|z_{1}\right|^{2}, \ldots,\left|z_{n}\right|^{2}$ and $\sum_{j=1}^{m}\left|z_{n+j}\right|^{2}$. This implies that the domain $\Omega_{a, k, n, m}$ is algebraic and complete Reinhardt. Moreover a little computing gives that $\sum_{j=1}^{n+m} z_{j} \frac{\partial \rho(z)}{\partial z_{j}}>$ 0 for $z \neq 0$. This shows that the domain $\Omega_{a, k, n, m}$ is smooth. Applying Lemma 1.3 to the case $f_{1}(z):=\left|z_{1}\right|, \ldots, f_{n}(z):=\left|z_{n}\right|, f_{n+1}(z):=\sum_{j=1}^{m}\left|z_{n+j}\right|^{2}$ and using the fact that the function $\alpha$ is strongly convex for $z \neq 0$, we deduce that the function $\rho$ is strongly convex for $z \neq 0$. This implies that $\Omega_{a, k, n, m}$ is strongly convex which completes assertion (1).

It is clear that $\Omega_{a, k, n, m} \subset D_{n, m}$. Now if $l \leq k$, then we have

$$
\begin{aligned}
N_{a, k}(z)^{2 l} & =\left(\sum_{\epsilon_{1}, \ldots, \epsilon_{n+1} \in\{-1,+1\}} \alpha_{\epsilon_{1}, \ldots, \epsilon_{n+1}}^{2 k}(z)+a^{2 k} \alpha^{2 k}(z)\right)^{\frac{l}{k}} \\
& \leq \sum_{\epsilon_{1}, \ldots, \epsilon_{n+1} \in\{-1,+1\}} \alpha_{\epsilon_{1}, \ldots, \epsilon_{n+1}}^{2 l}(z)+a^{2 l} \alpha^{2 l}(z)=N_{a, l}(z)^{2 l}, \quad z \in \mathbb{C}^{n+m} .
\end{aligned}
$$

Thus for $l \leq k$ we have $N_{a, k} \leq N_{a, l}$; consequently $\Omega_{a, l} \subset \Omega_{a, k}$. We observe that

$$
\left|z_{1}\right|+\cdots+\left|z_{n}\right|+\left|z_{n+1}\right|^{2}+\cdots+\left|z_{n+m}\right|^{2}=\lim _{k \rightarrow \infty} N_{a, k}(z) \quad \text { for } \quad z \in D_{n, m} .
$$

It follows that $D_{n, m}=\bigcup_{k=1}^{\infty} \Omega_{a, k, n, m}$. By Ramadanov's theorem [Ra] we see that the sequence of Bergman kernel functions $K_{\Omega_{a, k, n, m}}(z, w)$ converges uniformly on compact sets of $D_{n, m} \times D_{n, m}$ to the Bergman kernel of $D_{n, m}$. Now assertion (2) follows from the Hurwitz theorem and Lemma 1.1. 


\section{Concluding REMARKS}

In Bo1, H. P. Boas showed that there exists $R>0$ such that the following domain is non Lu Qi-Keng:

$$
\Omega:=\left\{(z, w) \in \mathbb{C}^{2}:|w|<\frac{1}{1+|z|} \&|z|^{2}+|w|^{2}<R^{2}\right\} .
$$

We can choose an exhaustion of $\Omega$ by the domains $\Omega_{k}$ defined by

$\Omega_{k}:=\left\{(z, w) \in \mathbb{C}^{2}:\left[|w|^{2 k}(1+|z|)^{2 k}+|w|^{2 k}(1-|z|)^{2 k}+\left(\frac{|z|^{2}+|w|^{2}}{R^{2}}\right)^{2 k}\right]^{\frac{1}{2 k}}<1\right\}$.

We see easily that the domains $\Omega_{k}$ are not only strongly pseudoconvex complete Reinhardt, diffeomorphic to the ball, but also algebraic.

We conclude this note by an open question which seems to be of interest.

Are all the domains $\Omega_{a, k, n, m}$ non-Lu Qi-Keng?

The author would like to thank E. H. Youssfi for proposing to him this problem as well as his various suggestions and indications during the preparation of this note.

\section{REFERENCES}

[Bo1] H. P. Boas, Counter example to the Lu Qi-Keng conjecture, Proc. Amer. Math. Soc. 97(2) (1986), 374-375. MR 87i:32035

[Bo2] H. P. Boas, The Lu Qi-Keng conjecture fails generically, Proc. Amer. Math. Soc. 124 (7) (1996), 2021-2027. MR 96i:32024

[BFS] Harold P. Boas \& Siqi Fu \& Emil J. Straube, The Bergman kernel function : Explicit formulas and zeroes, Preprint, 1997. CMP 98:01

[HLP] G. Hardy \& J. E. Littlewood \& G. Polya, Inequalities, Second Edition, Cambridge Mathematical Library (1994).

[HP] K. T. Hahn \& P. Pflug, On a minimal complex norm that extends the euclidian norm, Monatsh. Math. 105 (1988), 107-112. MR 89a:32031

[Lu] Qi-Keng Lu, On Kähler manifolds with constant curvature, Chinese Math. 8 (1966), 283298.

[PY] P. Pflug \& E. H. Youssfi, The Lu Qi-Keng conjecture fails for strongly convex algebraic domains, Arch. Math. 71 (1998), 240-245. CMP 98:16

[Ra] I. Ramadanov, Sur une propriété de la fonction de Bergman, C. R. Acad. Bulgare Sc. 20 (1967), 759-762. MR 37:1632

[SY] N. Suite \& A. Yamada, On the Lu Qi-Keng conjecture, Proc. Amer. Math. Soc. 59 (1976), 222-224. MR 54:13142

Université de Provence, LATP U.M.R C.N.R.S 6632, C.M.I, 39, Rue Joliot-Curie, 13453 Marseille Cedex 13, France

E-mail address: vietanh@gyptis.univ-mrs.fr. 